\title{
Les Helminthes du bétail et du porc dans la Fédération de Malaya ${ }^{(")}$
}

\author{
par J. EUZEBY \\ Professeur à l'École Vétérinaire de Lyon
}

\section{INTRODUCTION}

Le Professeur A. Raillet écrivait. en 1921, que l'Indochine est un paradis pour les parasites. Ce qui est vrai pour l'Indochine devait probablement l'être aussi pour la tres voisine Fédération de Malaya et. de fait, les Services Vétérinaires de ce pays mettaient depuis longtemps sur le compte de l'infestation et de l'infection parasitaires, l'état souvent médiocre de leur cheptel. C'est la raison pour laquelle le gouvernement de la Fédération avait demandé à l'Organisation des Nations Unies pour l'Agriculture et l'Alimentation (O.A.A.) l'assistance technique d'un expert, dont la tâche devait avoir.le double objet :

$1^{0}$ d'identifier les parasites des animaux domestiques et d'évaluer leur part de responsabilité dans l'affaiblissement du cheptel;

$2^{\circ}$ de former aux recherches parasitologiques un ou plusieurs membres des Services Vétérinaires locaux de façon à les rendre aptes à poursuivre les investigations après le départ de l'expert.

Sollicité par l'O.A.A. et ayant accepté la mission, qui m'était proposée dans le cadre du Programme Elargi d'Assistance Technique prévue par cette Organisation, j'ai passé six mois dans la Fédération de Malaya et me suis efforcé de répondre aux besoins exprimés par nos confrères de Malaisie.

La duree de mon séjour étant trop courte pour épuiser complètement la matière de la Mission qui m'était confiée, il m'a fallu limiter le champ de mon activité et, en accord avec nos confrères britanniques, responsables des S.V. Malayens et l'administration de l'O.A.A. j'ai limité le champ de mes investigations au domaine des helminthes des Ruminants et du Porc, qui nous paraissait être le probleme le plus important.
J'ai exposé ailleurs (1) un certain nombre d'informations diverses que j'ai pu recueillir au cours de mon séjour en Malaisie; sans y revenir ici. je voudrais seulement exposer les raisons qui font jouer aux helminthes un rôle aussi important dans la pathologie du bétail de ce pays. La fédération de Malaya, qui correspond, géographiquement, à la péninsule malaise ou presqu'île de Malacca s'étend entre $1^{0}$ et $7^{\circ}$ de latitude nord et $100^{\circ}$ et $105^{\circ}$ de longitude est; elle est donc située en pleine zone équatoriale. Les principaux caractères de son climat sont une température uniforme, de copieuses chutes de pluie et une forte humidite. On a l'habitude de diviser l'année en deux saisons de mousson' : mousson sudest et mousson nord-est, correspondant grossièrement à l'été et à l'hiver des pays tempérés; en réalité, à l'exception de la côte orientale, les différences de climat associées à l'idée de " mousson " sont à peine perceptibles en Malaya. Les mois situés entre ces deux « saisons » et qui correspondent théoriquement à nos printemps et automne, sont les plus humides de l'année dans l'ensemble du pays, mais les régions côtières ont leurs propres saisons de pluies. Encore qu'elles varient considérablement d'une région et d'une année à d'autres, les pluies atteignent une moyenne annuelle de $2,50 \mathrm{~m}$ et, dans la zone la plus humide, dépassent $4 \mathrm{~m}$. Aussi n'est-il pas étonnant que le degré hygrométrique soit très élevé, dépassant parfois 90 pour 100 !

Dans cette région, de type climatique « équatorialocéanique $\%$, la température oscille, dans les plaines, entre $24^{\circ}$ et $33^{\circ}$ tout au long de l'année.

(*) Article extrait d'un rapport de mission actressé à l'Organisation des Nations Unies pour l'Alimentation et l'Agriculture et publié avec l'accord' de cette Organisation.

(1) Mission en Malaya. Rev. Méd. Vét. (1956), 107 (6), 386-403 
On peut alors comprendre avec quelle exubérance, la plupart des helminthes peuvent se développer en ce pays où la chaleur et l'humidité élevées et régulières favorisent singulièrement à la fois les formes larvaires libres dans le milieu extérieur et, le cas échéant, la plupart des animaux hôtes intermédiaires.

Ceci posé, j'ai donc étudié la faune helminthologique chez les animaux suivants :

\section{Buffle : Bubalus bubalis \\ Zébu : Bibos indicus. \\ Chèvre : Capra hircus. \\ Porc : Sus scrofa domesticus.}

Je ne me suis pas attaché à l'étude du mouton car, en règle générale, les animaux de cette espèce ne sont pas élevés en Malaya, mais importés d'Australie d'où ils sont immédiatement dirigés vers les abattoirs. Seuls quclques individus provenant de l'Etat de Kélantan (au nord-est de la péninsule) où est entretenue une petite population ovine indigène ont fait l'objet de mon examen.

Le travail que j'ai accompli a été conduit selon le plan ci-après :

I) Étude statistique : basée sur de nombreuses autopsies et sur des examens hématologiques et coprologiques, quantitatifs et qualitatifs (coprocultures).

II) Étude biologique : dont le but était de préciser la chronologie évolutive des stades inférieurs des helminthes, en dehors de l'organisme de leurs hôtes.

III) Étude critique : destinée à montrer la distribution géographique des helminthes, l'importance de l'infestation générale, et l'incidence pathogène des vers parasites sur les animaux étudiés.

IV) Étude pratique : comportant l'exposé des règles de lutte anti-helminthique applicables au cheptel malayen.

Je ne rapporterai ici que les résultats de la partie statistique de mon travail et dégagerai seulement, ensuite, quclques considerations sur la place que tiennent les helminthoses dans la pathologie vétérinaire en Malaya et sur les mesures essentielles de leur prophylaxie.

\section{MATÉRIEL ET MÉTHODES}

Jail effectué mes recherches dans les laboratoires idu Veterinary Research Institute de la Fédération ,(V.R.I.), installé à Ipoh, capitale de l'Etat de Perak. Dans ces laboratoires, très modernes, très bien - équipés et très confortables (agréablement climatisés par une installation fournissant de $l^{3}$ " air con"ditionné ") j'ai trouvé toutes les facilités désirables upour mener à bien le travail qui m'était confié.
A) Les animaux examinés provenaient :

$1^{\circ}$ des abattoirs d'Ipoh,

$2^{\circ}$ du V.R.I., où ruminants et porcs sont entretenus en vue d'études expérimentales,

$3^{\circ}$ des abattoirs et des fermes dos divers Etats de la Fédération.

Sous ce rapport, un très gros effort a été fait par la Direction Génerale et les agents locaux des Services Vétérinaires pour faire parvenir au laboratoire les viscères et organes nécessaires à mes investigations. Ces organes et viscères étaient expédiés dans des seaux semblables à ceux utilisés pour la récolto du latex d'hévéa, immergés dans un liquide conservateur. En ce qui concerne les tubes digestifs, ils étaient divisés en portions ouvertes longitudinalement et plongées avec leur contenu dans le liquide fixateur, une solution aqueuse (1) de formol à $5 \%$. Pour les grands ruminants, on utilisait généralement: un seau pour la caillette, deux ou trois seaux pour l'intestin grêle, un seau pour le caecum, un seau pour le colon el le reclum; pour les petits ruminanls et le porc : un seau pour la caillette (ou l'estomac), un seau pour l'intestin grêle, un seau pour le gros intestin. Ainsi pendant plusieurs mois, les magasins de la gare d'Ipoh ont-ils été encombrés par des quantités inaccoutumées de seaux à latex en provenance des diverses régions de Malaya!

B) Les matières fécales, prélevées par les Vétérinaires de district, étaient contenues dans des flacons de verre et expédiées dans des bacs à glace par avion, les services de la Malayan Airways desservant régulièrement et rapidement les principales villes de la Fédération.

Tous les prélèvements étaient accompagnés d'un bordereau d'envoi mentionnant l'espèce de l'animal qui les avait fournis, son âge, son état général et, le cas échéant, les thérapeutiques anthelmintiques auxquelles l'individu avait pu être soumis.

En ce qui concerne les méthodes de rácolte, traitement et montage des vers, je ne les exposerai pas car j'ai suivi les règles classiques. Je préciserai seulement qu'aprè's essais de divers milieux c'est la gomme au chloral qui m'a paru le liquide de montage le plus simple et le plus pratique pour assurer une bonne conservation des préparations microscopiques dans le pays tropical où j'opérais; les quelques inconvénients que peut présenter ce milieu étaient palliés par la possibilité de maintenir les collections en atmosphère conditionnée.

Quant à la coprologie, j'ai utilisé les techniques suivantes :

(1) Solution aqueuse et non solution physiologique car la récolte des petits helminthes du tractus gastro-intestinal se faisant par sédimentation du contenu des viscères... et de celui des seaux. la présence de chlorure de sodium dans le liquide conservateur aurait gêné l'opération. 
a) Techniques qualitatives:

10 Chez les ruminants :

- méthode d'enrichissement par l'eau salée à

$15 \%$ (Cauchemez) pour les œufs de Nématodes:

- methode à l'iodo-rnercurate de potassium pour

les ceufs de Trématodes (Janeckso et Urbaneck);

$2^{\circ}$ Chez le porc

- méthode de Teleman;

- méthode par flottation avec usage d'une solution saturée de sulfate de magnésie.

b) Techniques quantitatives:

10 Méthode de Stoll : chez toutes les espèces, pour les numérations globales:

$2^{0}$ Méthode de Stoll et Loughlin pour la numération des ceufs de Trématodes du bétail.

Enfin pour les coprocultures et la récolte des larves infestantes de Nématodes, dont l'examen est nécessaire à l'identification rigoureuse des espèces. j'ai suivi les techniques courantes : culture en boite de Pélri sur papier filtre et récolte des larves au moyen de l'appareil de Baerman.

\section{RÉSULTATS STATISTIQUES}

J'exposerai successivement ces résultats, chez le zébu et le buffle, chez la chèvre (et, incidemment, le mouton) et chez le porc.

\section{$1^{\circ}$ Zébu et Buffle}

Les vers parasites que j'ai rencontrés appartiennent aux groupes et espèces suivants :

I. - Tractus digestif :

A) Nématodes.

- Neoascaris vitulorum (très fréquent chez les jeunes veaux zébus).

Strongyloides papillosus (intestin grêle).

- Esophagostomim (= Bosicola) radiatum. (larves : intestin grêle et colon)

(adultes : colon).

- Agriostomum vryburgı (colon).

Je dois ici faire observer que, contrairement aux indications fournies par les ouvrages classiques, je n'ai jamais trouvé ce ver dans le duodénum, localisation habituelle des Ancylostominae, mais toujours, très régulièrement, fixé à la muqueuse du colon.

- Bunostomum phlebotomum (intestin grêle).

- Gaigeria pachyscelis (intestin grêle).

En véritć, G. pachyscelis est très rare chez les grands ruminants de Malaisie, je ne l'ai trouvé, et très peu souvent, que chez le zébu, jamais chez le buffle.

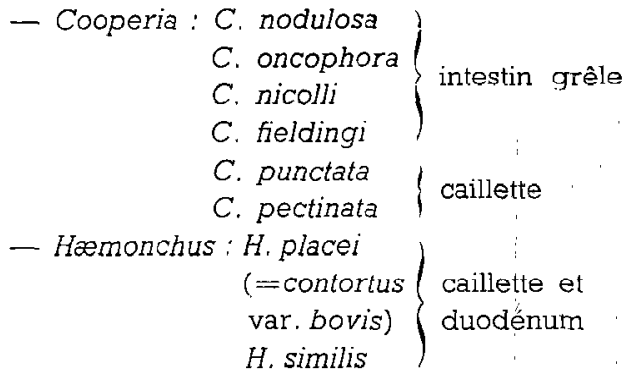

- Mecistocirrus: $M$. digitatus caillette.

Ce nématode, très commun, ressemble beaucoup, à l'cil nu, à Hæmonchus contortus et les femelles ont, en commun, le caractère morphologique, qui les fait appeler "vers mirlitons 》. Mais l'examen microscopique permet facilement la diagnose, basée principalement sur la longueur des spicules du mâle et l'absence de prolongement linguiforme supravulvaire des femelles dans l'espèce $M$. digitatus.

$$
\begin{array}{cl}
\text { - Trichuris: T. discolor } & \text { (caecum). } \\
\text { - Capillaria : C. bovis } & \text { (intestin grêle). } \\
\text { Capillaria sp. (caillette). }
\end{array}
$$

J'ai, en effet, trouvé plusieurs fois, dans la caillette du zébu une espèce du genre Capillaria dont, en l'absence de mâles, je n'ai pu faire l'identification précise. Les femelles que j'ai étudiées avaient tous les caractères de l'espece C. bovis, dont seule la localisation gastrique, très inhabituelle, les distinguait.

\section{B) Cestodes.}

- Avitellina : A. centripunctata (intestin grêle)

- Moniezia : $M$. expansa (intestin grêle).

C) Trématodes.

- Paramphistomum: P. cervi (rumen, réseau, caillette, duodénum).

- Cotylophoron: C. cotylophorum (mêmas localisations).

- Homalogaster : H. paloniæ (colon)

- Schistosoma: S. spindale.

Bien qu'il s'agisse, ici, d'un trématode parasite des vaisseaux, je signale dès maintenant son existence. En effet, ce ver détermine la formation sur le colon de taches hémorragiques au niveau desquelles il est facile de mettre en évidence les ceufs si caractéristiques de $S$. spindale. L'infestation due à cet helminthe est très fréquente et quasi constante chez los animaux provenant des Etats rizicoles de Malacca et de Kedah. D'autre part (et j'y reviendrai) j'ai aussi trouvé des schistosomes adultes dans la lumière même du colon.

II. - Foie :

Trématodes.

- Fasciola : F. hepatica.

F. gigantica. 
De ces deux espèces dont la diagnose, facile, repose sur les plus grandes dimensions et l'effacement de l'élargissement « scapulaire " de la seconde, $F$. gigantica est beaucoup plus répandue que $F$. hepatica.

\section{- Paramphistomum : P. explanatum.}

Cette espèce, qu'on a plutốt tendance, aujourd'hui, à désigner sous le nom de Gigantocotyle explanatum (qui fait allusion au grand développement de son acetabulum) se trouve comme les deux précédentes, dans les canaux biliaires. Elle m'a paru beaucoup moins fréquente que les autres « douves» du foie, et que ses « scurs 》 des réservoirs gastriques.

\section{III. - Pancréas :}

\section{Trématodes.}

- Eurytrema : E. pancreaticum (canaux pancréatiques).

Cette espèce est souvent si abondamment représentée que les individus s'échappent hors des canaux pancréatiques et passent dans l'intestin grêle. II n'est pas rare, d'autre part, qu'au moment de l'éviscération les douves du pancréas soient répandues dans la cavité péritonéale et se déposent sur le mésentère où, de par leur couleur rouge vif, elles simulent un semis de taches hémorragiques.

IV. - Cavité péritonéale :

$$
\begin{aligned}
& \text { Nématodes. } \\
& \text { - Setaria : S. cervi. } \\
& \text { S. digitata. }
\end{aligned}
$$

Ces deux espèces sont essentiellement différenciées par l'aspect de l'extrémité postérieure des femelles, présentant, chez $S$. cervi un petit bouton terminal pourvu d'épines, tandis que le bouton caudal est lisse chez la femelle de $S$. digitata. Nous discuterons plus loin l'importance de $S$, digitata pour la pathologie.

\section{V. - Appareil respiratoire:}

Nématodes.

- Dictyocaulus : D. viviparus : trachée et grosses bronches.

Cette espèce m'a paru relativement rare.

VI. - Appareil vasculaire :

\section{A) Trématodes.}

- Schistosoma spindale (veines mésentériques).

Ce trématode, parasite des veines mésentériques, très fréquent chez la plupart des zébus et des buffles, détermine la formation de taches hémorragiques sur la muqueuse colique, avec, çà et là, des ulcérations.
J'ai trouvé plusieurs fois, dans la lumière même du colon, des représentants de cette espèce, parfaiternent normaux et accouplés. Peut-être ces individus avaient-ils pu afteindre cette localisation anormale à la faveur des solutions de continuité de la muqueuse, provoquées par l'action traumatique et toxique des œufs.

\section{B) Nématodes.}

- Elæophora : E. pœli (aorte de buffle).

L'extrémité antérieure, très effilée, de la femelle s'enfonce dans la paroi de l'artère, où elle provoque la formation de nodules inflammatoires du volume d'un pois à celui d'une noisette; le reste du corps flotte librement dans la lumière du vaisseau.

\section{- Onchocerca: O. armillata (aorte du buffle et} du zébu).

Ce ver, entièrement logé dans la pároi aortique dont il est très difficile de l'extraire intégralement, apparaît sous l'aspect de minces lignes spiralées, de couleur verdâtre, dispersées dans les portions antérieures de l'aorte.

\section{$2^{\circ}$ Petits ruminants : Chèvre (et, occasionnellement, Mouton)}

En règle générale, on retrouve, chez les petits ruminants, des especes identiques à celles du buffle et du zébu, ou des espèces voisines. Cependant, certaines d'entre elles sont plus spécifiques de tel ou tel hôte.

J'ai trouvé, chez la chèvre, les helminthes ciaprès :

I. - Tube digestif :

A) Nématodes.

- Strongyloïdes papillosus (intestin grêle).

- Esophagostomum (= Protoracrum) colum bianum.

- Esophagostomum (= Hysteracrum) venulosum.

Ces deux espèces dans le colon et le caecum.

- Gaigeria pachyscelis (intestin grêle).

Cette espèce, rarissime chez le zébu, est extrêmement fréquente, pratiquement constante, chez la chèvre. Je l'ai aussi observée chez quelques-uns des moutons indigènes que j'ai eu l'occasion d'autopsier.

- Bunostomum trigonocephalum (intestin grêle).

Je n'ai rencontré $B$. trigonocephalum que chez les moutons provenant de l'élevage local de l'Etat de Kelantan. Je ne l'ai jamais observé chez la chèvre. malgré l'examen de très nombreux intestins. 
- Hæmonchus contortus (caillette, duodénum).

- Cooperia oncophora (intestin grêle).

- Trichostrongylus : T. probolurus : duodénum.

T. colubriformis : duodenum.

T. axei (= extenuatus) (1) : caillette et duodénum.

$T$. vitrinus : duodénum.

J'ai trouvé fréquemment la plupart de ces espèces de Trichostrongyles chez la chèvre, alors que je n'en ai pas rencontré chez les grands ruminants. Toutefois, en ce qui concerne T. axei, cet helminthe me paraît plus rare et, en tout cas, beaucoup moins commun que les autres espèces du même genre.

- Ostertagia circumcincta: caillette et intestin grêle.

Cette espèce est la seule que j'aie pu observer dans le genre Ostertagia. Je ne l'ai, d'ailleurs, rencontrée que très rarement et seulement chez la chèvre.

- Trichuris : T. ovis : cæoum.

T. globulosa: cæcum (deux fois seulement).

- Capillaria : C. longipes.

C. brevipes : intestin grèle.

B) Cestodes.

- Moniezia expansa : intestin grêle (en quantité parfois prodigieuse)

C) Trématodes.

- Paramphistomum cervi.

- Cotylophoron cotylophorum : rumen, réseau, caillette et duodénum.

II. - Foie :

- Fasciola hepatica.

- F. gigantica : canaux biliaires.

III. - Pancréas :

- Eurytrema pancraticum.

IV. - Appareil respiratoire :

- Dictyocaulus filaria.

Bien que plus fréquente (à mes yeux) que $D$. viviparus chez le zébu, cette espèce me paraît relativement rare.

\section{$3^{\circ}$ Pore}

I. - Tractus digestif :

Nématodes.

- Ascaris suum: intestin grêle

(1) Quelques auteurs n'admettent pas l'identité de T. axei et T. extenuatus, En vérité ces deux espèces sont si voisines que, dans cette étude essentiellement pratique, on peut les considérer comme identiques.
Espèce très fréquente, déterminant souvent des infestations massives.

- Esophagostomum dentatum: colon.

- Globocephalus : G. urosubulatus.

G. samoensis : intestin grêle.

- Trichuris trichiura: cæcum.

- Physocephalus sexalatus : estomac.

- Gnathostoma hispidum: estomac.

Cette espèce m'a paru moins commune que la précédente.

\section{II. - Reins :}

Nématodes.

- Stephanurus dentatus.

Cette espèce est surtout logée dan's le tissu adipeux péri-rénal, où elle est très fréquente. On la trouve aussi dans le bassinet et les uretères.

III. - Appareil respiratoire:

Nématodes.

- Metastrongylus : $M$. elongatus (= apri).

M. salmi : grosses et moyennes bronches.

\section{4' Helminthes divers}

Outre les espèces mentionnées dans la nomenclature que je viens d'exposer, j'ai pu obtenir un certain nombre d'informations concernant d'autres helminthes du bétail et du porc :

- Onchocerca gibsoni : est tout à fait commun sous la peau du zébu, où il provoque la formation de lésions nodulaires.

- Cysticercus cellulosæe et C. bovis formes larvaires des "vers solitaires 》 de l'homme, Tænia solium et $T$. saginata) existent chez le porc et le zébu. Le premier est, comme partout, beaucoup plus rare que le second.

- Echinococcus granulosus (= Tænia echinococcus), existe, sous sa forme larvaire ( $E$. polymorphus) chez les ruminants et le porc.

- Trichinella spiralis semble ne pas exister en Malaya. La trichinoscopie n'étant pas pratiquée, il est difficile d'être très affirmatif en cette matière, mais les rapports du Service de Santé de la Fédération ne signalent pas la Trichinose humaine, bien que, à défaut des Malais (musulmans), les Chinois et les Indiens soient volontiers consommateurs de porc.

\section{DISTRIBUTION ET FREQUENCE DES HELMINTHES}

En ce qui concerne la distribution géographique, la répartition et la fréquence des parasites dont je viens de donner la liste, on peut dire que tous peuvent être observés dans les divers Etats de la Fédération, avec, cependant, une fréquence assęz inćgale. 
$1^{\circ}$ Certains d'entre eux sont des parasites quasi constants affectant pratiquement $100 \%$ des animaux, de façon plus ou moins importante.

Il en est ainsi pour les espèces suivantes :

- Ascarides du porc et, un peu moins fréquemment, du veau,

- Hæmonchus des grands et petits ruminants,

- Trichostrongylus colubriformis chez la chèvre,

- Cooperia punctata chez tous les ruminants,

- Mecistocirrus digitatus chez le zébu,

-- Gaigeria pachyscelis et Esophagostomes ( $O$. columbianum et $O$. venulosum) chez la chèvre

- Fsophagostomes, Globocephales et Stephanures du porc,

- Elæophora pceli chez le buffle,

-- Paramphistomidæ des réservoirs gastriques et Eurytrema des canaux pancréatiques chez le zébu et la chèvre.

$2^{\circ}$ La plupart des autres helminthes mentionnés dans les listes ci-dessus sont tous très largement répandus, sans avoir le caractère de quasi constance des précédents.

$3^{\circ}$ D'autres espèces miont, au contraire, paru beaucoup plus rares.

- Gnathostoma hispidum de l'estomac du porc,

- Paramphistomum explanatum des canaux biliaires des grands ruminants,

- Dictyocaulus viviparus et $D$. filaria.

$4^{\circ}$ Enfin, il est des espèces banales en France et que j'ai eu la surprise de ne jamais rencontrer; il s'agit, en particulier, de :

- Dicrocoelium lanceolatum.

-. et des Protostrongylinæ des petits ruminants.

Peut-être cette absence peut-elle s'expliquer par le caractère xérophilique des mollusques hôtes intermédiaires de ces vers, auxquels le climat très humide de Malaya ne convient pas.

\section{INCIDENCE DES INFESTATIONS VERMINEUSES SUR LA PATHOLOGIE DU BÉTAIL ET DU PORC EN MALAYA}

Je ne ferai pas, ici, l'étude particulière de chacun des helminthes susceptibles de parasiter les animaux envisagés, mais j'exposerai seulement les points les plus saillants et les plus dignes de remarque.

\section{A) Chez les ruminants :}

- L'ascaridiose des jeunes bovidés est un problème de la plus haute importance chez le zébu comme chez le buffe; les animaux de 2 à 3 mois sont très infestés et la mortalité atteint chez eux jusqu'à $30 \%$ des individus. D'autre part, à l'infestation ascaridienne est souvent liée la colibacillose du veau.
Des observations précises faites à la Station d'Elevage de Kluang (Johore) ont montré que le traitement anti-ascaridien diminue considérablement les cas de " white scour ».

- Les « strongles » hématophages du tube digestif sont, de par leur fréquence, des causes importantes d'anémie parasitaire.

A côté des Trichostrongylidæe' (Hæmonchus sp., Mecistocirrus digitatus), certains Ankylostomidæ sont très pathogènes à ce point de vue, particulièrement Bunostomum phlebotomum, que j'ai vu plusieurs fois par milliers sur des animaux arrivés au dernier stade d'une anémie intense.

Par contre, Agriostomum vryburgi (dont j'ai déjà signalé la localisation colique), beaucoup moins fréquent, est aussi beaucoup moins pathogène.

- Les fascioloses hépatobiliaires ont, en Malaya, la gravité qu'elles affectent sous toutes les latitudes. Mais, tout en intéressant la plupart des régions de la péninsule, elles posent surtout un problème dans les territoires particulièrement humides, des Etablissements de Malacca et Penang et l'Etat de Kedah.

- L'affection à $S$. spindale extrêmement banale chez les animaux des régions rizicoles, ne m'a pas paru être particulièrement grave, malgré l'étendue parfois considérable des lésions hémorragiques qu'elle détermine sur le colon.

Au contraire :

- La question du rôle pathogène de Eurytrema pancraticum est très discutée par nos confrères de Malaisie. Certains rendent responsable ce parasite de certains cas d'un curieux syndrome, à étiologie encore imprécise, la « $R$. disease » survenant chez le zébu (1). Il est, on effet, possible que des douves puissent obstruer les canaux excréteurs du pancréas et provoquer, par défaut de suc pancréatique, une mauvaise digestion des protéines et lipides de la ration. Mais combien de fois peut-on observer des infestations massives à $E$. pancreaticum sur des animaux en très bon état! A mon avis, ce parasite est, le plus souvènt, très peu pathogène.

- Il est très intéressant de signaler en Malaya l'existence de la " filaire " Setaria digitata. On sait, depuis les très intéressants travaux de Chusaburo Shoho et $C$. Innes la part active que prennent les larves de ce parasite dans le déterminisme d'un syndrome nerveux, affectant la chève et le cheval. Ce syndrome étudié en Corée, au Japon, à Ceylan (où il est connu sous le nom de " kumry ») est dû à la migration dans le système nerveux central, de larves de Setaria digitata inoculées par les moustiques

(1) On trouvera une étude préliminaire de ce syndrome dans la publication de MM. C. C. Renwick et R. S. T. Bowden, R.F. Couglan et A. G. Brooke, J. of the Malayan Vet. Med. Ass. 1956, 1 (2), $32-41$. 
à des hôtes anormaux. Je n'ai pas eu le temps d'enquêter à ce sujet pendant mon séjour en Malaya, mais, puisqu'existe le parasite adulte chez les bovidés, il serait bien improbable que le syndrome dû aux larves égarées chez des animaux autres que leurs hôtes naturels ne se manifeste pas.

\section{B) Chez le porc :}

- L'ascaridiose constitue, à mes yeux, la plus sérieuse des infestations porcines et elle revêt chez le porc le même caractère de gravite que chez les bovidés.

- Après elle, la Métastrongylose paraît mériter une particulière mention, non seulement par les accidents dus à l'obstruction mécanique des moyennes bronches, mais aussi par les phénomenes d'allergie focale liés aux larves qui envahissent le parenchyme. Beaucoup de poumons de porcs examinés présentent une réaction inflammatoire cedémateuse, reconnaissant cette étiologie.

- Par contre, il ne m'a pas paru que les Esophagostomes et Globocephales, malgré leur présence en quantité partois importante, aient, à eux seuls, un pouvoir pathogène considérable.

- Enfin, Stephanurus dentatus, bien que ne provoquant pas des troubles graves sur le plan médical est un helminthe redoutable du point de vue economique. Sa très grande fréquence est cause de la saisie de la plus grande partie des reins et de la graisse périrénale et il en résulte à la longue des pertes élevées.

Les lignes qui précèdent ne prétendent pas donner une vue complète de la pathologie liée aux infestations vermineuses parmi les mammifères domestiques du cheptel de Malaya; en réalité, il aurait fallu discuter le rôle pathogène de chacur des helminthes en en dégageant tous les aspects, ce qui n'était pas possible dans le simple aperçu que j'en ai voulu donner.

J'ajouterai seulement, pour conclure ce chapitre, que $s i$, à l'exception de quclqucs ontités réellement meurtrières (ascaridioses, fascioloses, "strongyloses » dues aux espèces hématophages), les helminthoses ne sont pas des maladies "spectaculaires". leur incidence générale sur l'état du cheptel de Malaya n'en est pas moins considérable. Ainsi se trouve confirmée l'opinion qu'en avaient toujours eue les Vétérinaires Britanniques et Malayens de la Fédération.

Il résulte de cette conclusion la nécessité de continuer et d'intensifier la lutte anthelminthique. Dans un pays comme la péninsule malaise, cette lutte comporte, sur le plan pratique comme sur le plan psychologique, des aspects très particuliers. I'ai tente dans le rapport adressé à l'O.A.A. d'en dégager les règles dont je ne peux, ici, que résumer l'essentiel, dans le but d'être utile à ceux de nos confrères des pays tropicaux qui pourraient se trouver dans des conditions semblables à celles des Vétérinaires de Malaisie.

\section{RÈGLES GENERALES DE CONTROLE DES HELMUNTHOSES EN MALAYA}

L'organisation de la lutte contre les helminthoses dépend des méthodes selon lesquelles est conduit l'élevage en Malaya et qui diffèrent beaucoup dans les Stations gouvernementales dirigées par les Services Vétérinaires et dans les petites exploitations privées.

\section{A) Dans les Centres gouvernementaux :}

$1^{\circ}$ L'élevage des Bovins est effectué selon les regles suivantes :

Les jeunes veaux de moins de 10 mois sont maintenus à l'étable, où un veau et sa mère occupent une stalle de 10 à $12 \mathrm{~m} 2$, désinfectée tous les mois.

Jusqu'à l'âge de 3 mois, les veaux sont allaités par leur mère. $\Lambda$ partir de 4 mois, ils reçoivent, outre le lait maternel, de l'herbe et des aliments concentrés; ils sont complètement sevrés à 10 mois.

On les conduit alors sur les pâturages où les jeunes zébus ne séjournent que pendant la journée et sont rentrés la nuit, tandis que les jeunes buffles y vivent " à temps plein ». Les parcs sont divisés en portions de 120 à 800 ares (1), où on laisse paître 100 veaux sur 40 ares pendant 2 jours : par exemple sur une surface de 120 ares on laissera 100 veaux pendant 6 jours, sur une surface de 800 ares le mềme nombre d'individus sera maintenu pendant 40 jours. A l'expiration de ce laps de temps, les animaux sont conduits sur une autre parcelle; la végétation qui demeurait sur la parcelle précédemment pâturée est coupée, le sol est hersé et le terrain mis en repos pendant 2 mois.

Les bovins adultes sont élevés dans des conditions assez semblables mais le nombre d'individus admis par 40 ares de surface est deux fois moindre.

$2^{\circ}$ En ce qui concerne les Chèvres, jusqu'à l'âge de 4 mois on laisse les jeunes avec leur mère, dans les locaux. Sevrés à 4 mois, les chevreaux sont conduits sur les pâturages de 9 heures à midi et de 2 heures à 5 heures. On ne leur affecte pas de parcs spéciaux, mais ils paissent librement sur les mêmes aires que les bovins.

Il n'est pas douteux que ce mode d'élevage soit très rationnel : la séparation sur les parcs des jeunes et des adultes previent de trop fortes infestations des premiers au contact des seconds. La rotation des pâturages, associée au hersage du sol, favorise

(i) Solt approximativement 3 à 20 acres anglais. 
la destruction des formes larvaires d'helminthes; la désinfection régulière des' locaux détruit de nombreux éléments vermineux. Il en résulte que les holminthoses ne sont pas, en règle gćnérale, un très gros problème dans les fermes gouvernementales. Toutefois l'ascaridiose demeure une importante maladie, surtout chez les jeunes buffles. Les cufs d'ascarides, très résistants aux agents chimiques ne sont détruits que par des moyens physiques (eau bouillante, etc.); d'autre part les veaux sont souvent infestés à partir de leur mère : infestation prénatale (par migration des larves infestantes dans les vaisseaux placentaires) ou infestation néonatale (par succion de mamelles souillées de débris fécaux renfermant des cufs infestants). Aussi ai-je proposé, pour compléter la prophylaxie applicable à l'ascaridiose, les mesures suivantes:

- désinfection bi-mensuelle des stalles par l'eau bouillante ;

- administration mensuelle de pipérazine aux femelles gestantes pour atteindre les larves en transit dans le tube digestif ;

- traitement mensuel des veaux jusqu'à l'âge de 8 mois, époque où leur réceptivité devient beaucoup moindre.

Enfin, pour éviter autant que -cossible les autres maladies vermineuses, telles que "Strongyloses 》 et teniasis, j'ai recommandé, associé à la rotation des pâturages, l'épandage de cyanamide calcique (120 kg à l'ha), bon engrais nitré et doué d'activité toxique sur les formes larvaires des « Strongles » et sur les acariens hôtes intermédiaires des Moniezia; le hersage consécutif à l'épandage de la cyanamide en favorise la dispersion.

B) Dans les petites exploitations privées, les conditjons sont loin d'être aussi favorables.

$1^{0}$ L'absence de prairies oblige les ruminants à paître où ils le peuvent, principalement le long des routes ou encore entre les arbres, dans les plantations de cocotiers ou d'hévéas. Ir en résulte, outre une nourriture très médiocre en qualité comme en quantité, l'impossibilité absolue d'organiser une prophylaxie rationnelle. Les seules mesures que l'on puisse profcser consistent en l'institution de traitements systématiques, selon un rythme périodique, dans le but de detruire les éléments d'infestation je plus tôt possible après leur pénétration chez les animaux et avant qu'ils aient pu exercer un rôle pathogène.

La périodicité de ces traitements est, évidemment, liée à la chronologie évolutive dos vers hors de l'organisme de leurs hôtes, c'est-à-dire au délai qui s'écoule entre l'expulsion par l'animal des œufs $d$ 'helminthes et à la formation, à partir de ces ceufs, d'éléments infestants. En Malaya, ce délai est d'en- viron 3 mois pour $F$. hepatica, 4 semaines pour $M$. expansa, 2 semaines pour $N$. vitulorum, 1 semainet pour les « Strongles».

Par conséquent, la prophylaxie médicale devrait comporter :

pour la fasciolose : un traitement trimestriel;

pour le teniasis : un traitement mensuel;

pour l'ascaridiose : un traitement bi-mensuel;

pour les « strongyloses 》 : un traitement hebdomadaire.

En réalité, pour les deux dernières affections, il n'est pas possible, pratiquemenl, de traiter les animaux avec une telle fréquence. Mais si l'on tient compte qu'il s'écoule une période de 10 à 25 jours depuis le moment de l'infestation jusqu'à celui où les parasites développeront leur action pathogène. on peut se contenter dans les « strongylóses ». et l'ascaridiose, comme dans le teniasis, d'ur traitement mensuel, compatible avec la rentabilité de l'operation.

$2^{\circ}$ En ce qui concerne l'élevage porcin, la prophylaxie des helminthoses ne m'a retenu que dans les exploitations privées, l'élevage du porc n'étant pas pratiqué dans les fermes d'État dépendant des Services Vétérinaires.

Les porcheries sont plus ou moins bien installées selon les régions. Dans la plupart des cas, elles sont petites, surpeuplées, et leur sol en terre battue n'en permet pas une désinfection rigoureuse à laquelle d'ailleurs, les propriétaires ne songent le plus souvent pas! Dans.les « Nouveaux villages 》 (agglomérations artificiellement créées pour rassembler les hameaux dispersés et les mettre à l'abri des raids terroristes), elles sont, au contraire, souvent très rationnellement construites, divisées en loges à sol cimenté et ne contenant, chacune, qu'un petit nombre d'animaux. Le nettoyage et la désinfection y sont donc faciles.

Dans les régions littorales, les porcheries sont parfois installées au-dessus de la mer et pourvues d'un plancher a claire-voie facilitant beaucoup le nettoyage. D'autre part, ce nettoyage peut être spontanément réalisé par la mer elle-même, au moment du flux.

Les deux helminthoses porcines dont la prophylaxie devrait, à mon avis, être systématiquement réalisée sont l'ascaridiose et lá bronchite vermineuse.

Pour l'ascaridiose, j'ai recommandé :

a) le traitement des truies gestantes, dans le but : 1o de détruire les larves en transit dans le tube digestif avant que ces larves n'aient entrepris leurs migrations, susceptibles de les mener dans les vaisseaux placentaires ; $2^{\circ}$ de prévenir chez les mères elles-mêmes des infestations qui en feraient 
des sources de parasites pour les jeunes porcèlets;

b) l'installation d'une stalle d'accouchement dans laquelle, au moment du part, la truie est conduite après avoir été soigneusement nettoyèe des débris fécaux et des débris de litière dont elle pourrait être souillée:

c) la " rotation bimensuelle des loges» : comportant, toutes les quinzaines, le passage de la truie et des porcelets dans une loge propre et désinfectée, ceci afin d'empêcher l'infestation possible des jeunes à partir des ceufs d'ascarides émis par la truie. Cette rotation serait à observer jusqu'à ce que les animaux aient atteint I'âge de 5 mois.

Quant à la Métastrongylose, la seule mesure prophylactique possible en Malaya m'a paru être l'administration régulière de phénothiazine dans le but de détruire les larves infestantes au cours de leur passage dans le tube digestif. Cette administration devrait être faite toutes les trois semaines.

$\mathrm{La}$ bronchite vermineuse affectant les porcs élevés en liberté, sur des terrains où ils ont la possibilité d'absorber les vers de terre hôtes intermédiaires des parasites, il est bien difficile d'organiser une prophylaxie générale basée sur la collecte et la stérilisation des matières fécales émises par les porteurs de Métastrongles.

\section{REMERCIEMENTS}

C'est pour moi un très agréable devoir que de remercier ceux qui m'ont permis d'accomplir el travail qui fait l'objet de cette publication.

Ces remerciements s'adressent d'abord à l'administration de l'O.A.A, et particulièrement aux responsables de la Branche de la Production Animale : Dr K.-V.-L. Kesteven, Sir Thomas Dalling, Dr N.-R. Reid, Dr C. Castrioty qui m'ont honoré de leur confiance en me proposant la mission d'expert parasitologue en Malaya et qui ont tout fait pour m'en faciliter l'exécution.

Mes confrères britanniques et malayens de la Fédération m'ont surabondamment prodigué loutes les marques de la plus parfaite amitié et se sont ingéniés à rendre mon séjour parmi eux non seulement utile mais très agréable.

Que tous en soient vivement et sincèrement remerciés, mais particulièrement : MM. W.-E. Tancaster, Directeur des Services Vétérinaires, C.-W. Wells, Directeur adjoint, R.-S.-T. Bowden, Chef du Laboratoire de Recherches d'Ipoh et aussi mon assistant M. C.-S. Shanta.

Mais je dois une gratitude toute particuliere à mes Chefs hiérarchiques, qui m'ont autorisé à accepter l'offre de I'O.A.A. : M. le Professeur Jung, Directeur de l'Ecole do Ĺyon, M. le Professeur Petit, Inspecteur Général des Ecoles Vétérinaires, et surtout $M$. le Professeur Vuillaume, Chef des Services Vétérinaires au Ministère de l'Agriculture : je suis heureux de l'assurer, ici, de mes sentiments très respectueusement reconnaissants.

\section{SUMMARY}

Helminths of cattle and pig in Malay.

Having stated the reasons which led to the study of helminthiasis of cattle and pigs in Malay and pointed out the plan of work and the methods followed, the author reports the results of his research. He would like to insist upon the statistical conclusions of his work. On the other hand, he gives only a simple outline of the general incidence of helminthiasis on the pathology of infested domestic mammals, and the rules of prophylaxy applicable, in the Malay Federation.

\section{RESUMEN}

Los helmintos del ganado y del cerdo en Malaya.

Después de exponer las razones que le han Ilevado a estudiar los helmintos del ganado y de los cerdos en Malaya, y de haber indicado el plan de trabajo y los métodos seguidos, el autor resume los resultados de sus investigaciones. Insiste, sobre todo, en las conclusiones estadísticas de su estudio. Por el contrario no da más que una simple referencia de la frecuencia de helmintiasis en la patologia de los mamíferos domesticos infestados, y de las reglas de profilaxis applicables en la Federación Malaya. 